(2) Open Access Full Text Article

\title{
Sequential Keraring implantation and corneal cross-linking for the treatment of keratoconus in children with vernal keratoconjunctivitis
}

This article was published in the following Dove Press journal:

Clinical Ophthalmology

24 October 2017

Number of times this article has been viewed

\author{
Mortada A Abozaid \\ Ophthalmology Department, \\ Faculty of Medicine, Sohag \\ University, Sohag, Egypt
}

Correspondence: Mortada A Abozaid Ophthalmology Department, Faculty of Medicine, Sohag University, Sohag, Egypt Tel +20932309244

Fax +20932325531

Email mourtada_abdelaal@med.sohag. edu.eg
Purpose: The purpose of this study was to assess the safety and efficacy of femtosecond laser-assisted Keraring implantation followed by transepithelial accelerated corneal collagen cross-linking (CXL) for the treatment of keratoconus in children with vernal keratoconjunctivitis (VKC).

Study design: This is a prospective interventional non-comparative case series.

Patients and methods: Eighteen eyes of 11 children with keratoconus and VKC were included in this study. All the cases were treated with femtosecond laser-assisted Keraring implantation followed after 2 weeks by transepithelial accelerated CXL, and the patients were followed up for 1 year.

Results: The preoperative mean uncorrected visual acuity (UCVA) was $1.01 \pm 0.2(\log M A R)$, whereas the postoperative mean UCVA was $0.6 \pm 0.2$. The preoperative mean best-corrected visual acuity (BCVA) was $0.6 \pm 0.1$, whereas the postoperative mean BCVA was 0.40 \pm 0.2 . The preoperative average keratometry was $50.3 \pm 2.7 \mathrm{D}$, whereas the postoperative average keratometry was $45.8 \pm 3.1 \mathrm{D}$.

Conclusion: The results of this study suggest that femtosecond laser-assisted Keraring implantation followed by CXL is safe and effective in the management of keratoconus in children with VKC. However, studies with a longer follow-up period are needed.

Keywords: cross-linking plus, intrastromal corneal ring segments, pediatric keratoconus, spring catarrh

\section{Introduction}

Keratoconus is a noninflammatory corneal disease with an annual estimated incidence of two in 100,000 and a prevalence of 54.5 in 100,000. It is a bilateral disease, although one eye may be affected before the other, characterized by conical shape of the cornea due to progressive thinning of its stroma. The corneal thinning induces myopia, irregular astigmatism, and high-order aberrations which lead to variable impairment of vision. ${ }^{1-3}$

The onset is usually at puberty with progression until the third to fourth decade of life, when it usually arrests. It may, however, commence later in life and progress or arrest at any age. The most common presentation of keratoconus is as an isolated sporadic disorder with no other associated systemic or ocular disease. However, it may be associated with conditions like Down syndrome, Leber's congenital amaurosis, and chronic eye rubbing (eg, vernal keratoconjunctivitis [VKC]). ${ }^{2}$

$\mathrm{VKC}$ is a severe form of ocular allergic conjunctivitis which affects boys more than girls with onset generally before the age of 10 years. Ninety-five percent of cases remit by the late teens although many of the remainder develop atopic keratoconjunctivitis. 
$\mathrm{VKC}$ is relatively common in warm dry climates such as the Mediterranean, sub-Saharan Africa, the Middle East, and the Indian subcontinent and is unusual in temperate regions. ${ }^{4,5}$

The modern management of mild to moderate cases of keratoconus incorporate two separate lines of treatment. The first line is the corneal collagen cross-linking (CXL) to strengthen the cornea and halt progression of the disease while the second line consists of visual rehabilitation through spectacles, contact lenses, or intrastromal corneal ring segments (ICRS).

CXL is a technique that depends on a photochemical reaction between ultraviolet $\mathrm{A}$ light and riboflavin (vitamin $\mathrm{B}_{2}$ ) within the corneal stroma with the development of chemical bonds between collagen fibrils, thus strengthening the cornea and slowing or stopping the progression of keratoconus and other corneal ectasias such as post-laser in situ keratomileusis ectasia. ${ }^{6,7}$

In children with progressive keratoconus, the epithelium-on (transepithelial) cross-linking is less painful and provides similar effectiveness and less complications than the epithelium-off procedure. ${ }^{8}$

Implantation of ICRS into a circular corneal tunnel improves visual acuity and reduces the central corneal steepening in ectatic corneas. ${ }^{9,10}$ Keraring is a type of ICRS that are made of polymethyl methacrylate and are available in different arc lengths. They are triangular in cross-section with a $600-\mu \mathrm{m}$ base and an apical diameter of $5 \mathrm{~mm}$. They are of variable thickness $(0.15,0.20,0.25,0.30$, and $0.35 \mathrm{~mm})$ in $0.05 \mathrm{~mm}$ steps. ${ }^{11,12}$ The introduction of femtosecond laser technology for ICRS implantation allowed the surgeon to program a corneal tunnel dissection at a predetermined depth with a very high degree of accuracy, thus avoiding the potential inaccuracies of mechanical dissection. ${ }^{13,14}$

Although many studies have been published on the management of pediatric keratoconus, only few dealt with children having VKC. The purpose of this study was to assess the outcome of femtosecond laser-assisted Keraring implantation followed by transepithelial accelerated CXL in children with keratoconus and VKC.

\section{Patients and methods}

Eighteen eyes of 11 children with keratoconus and VKC were recruited for this prospective interventional non-comparative study in the period between January 2015 and April 2016 at the Ophthalmology Department of Sohag University Hospital in collaboration with Sohag Future Femtolasik center in Egypt. The study followed the Declaration of Helsinki, and an approval was obtained from the ethics committee of Sohag Faculty of Medicine. Written informed consent was obtained from the parents of all children after explanation of the risks and benefits of these procedures.

All the patients were $<18$ years old, had progressive keratoconus (defined as $1 \mathrm{D}$ increase in steepest keratometric reading over a 6 -month period), poor $\mathrm{BCVA}(<6 / 18$, ie, $<0.48 \log$ MAR), clear central cornea, and corneal thickness being $>450 \mu \mathrm{m}$ at the planned incision sites.

The exclusion criteria included patients with severe form of VKC (as evidenced by conjunctival granulomas, pannus, shield ulcer and/or limbal stem cell deficiency with conjunctivalization) and eyes with corneal haze or opacity, severe keratoconus (keratometric values of $>65.0 \mathrm{D}$ ), active ocular infection or inflammation or previous surgery (eg, collagen cross-linking).

Preoperative assessment of the patients included uncorrected (UCVA) and best-corrected visual acuity (BCVA), manifest and cycloplegic refraction, slit lamp, and fundus examination in addition to intraocular pressure measurement. Diagnosis and grading of keratoconus were done using a Sirius Scheimpflug imaging camera (CSO, Italy).

\section{Surgical procedure}

The Keraring nomogram provided by the manufacturer, Mediphacos, was used to select the proper ring segments, and all cases were operated on by one surgeon. The Keraring implantation was done under general anesthesia while the cross-linking was performed under topical anesthesia. Patients with active $\mathrm{VKC}$ are given medical treatment, and surgery was delayed till the allergic episode subsides completely.

1. Femtosecond laser-assisted Keraring implantation: Before starting general anesthesia, the patient is asked to look at the flashing light point coming from the microscope head to mark the center of the cornea. Then and after induction of general anesthesia, the suction ring is applied and the corneal tunnel is created with femtosecond laser (iFS Advanced femtosecond laser; Abbott Laboratories, Abbott Park, IL, USA). The parameters of the tunnel were as follows: inner diameter $5 \mathrm{~mm}$; outer diameter $5.9 \mathrm{~mm}$; depth $75 \%$ of the thinnest central corneal thickness; incision site at the axis of $\mathrm{K} 2$ (the steepest corneal meridian). Then, patency of the tunnel is ensured with a spatula passed through it followed by implantation of the Keraring segments. A contact lens is applied and left for 3 days and eyedrops (steroids and antibiotics) are prescribed for 2 weeks.

2. Transepithelial accelerated CXL was done for all cases 2 weeks after the initial ring implantation procedure under topical anesthesia with benoxinate $0.4 \%$ Benox $^{\circledR}$; Epico, Inc., Cairo, Egypt) given three to five times every 5 minutes. 
Dextran-free hypo-osmolar riboflavin drops containing benzalkonium chloride to enhance epithelium permeability (ParaCel; Avedro, Waltham, MA, USA) are dripped onto the cornea every 1.5 minutes for 4.5 minutes, and then benzalkonium chloride-free riboflavin drops (VibexXtra; Avedro) are dripped every 1.5 minutes for 6 minutes. This was followed by 2 minutes and 40 seconds of accelerated CXL (The KXL accelerated CXL System; Avedro) using the pulsed mode ( 2 seconds on $/ 1$ second off) with a power of $45 \mathrm{~mW} / \mathrm{cm}^{2}$ and a total energy of $7.2 \mathrm{~J} / \mathrm{cm}^{2}$ irradiated. A contact lens is then applied and left for 1 week.

\section{Postoperative treatment}

The topical treatment included the antibiotic moxifloxacin (Vigamox; Alcon Laboratories, Inc., Fort Worth, TX, USA), the steroid prednisolone (PredForte; Allergan, Inc., Irvine, CA, USA), and artificial tears (Systane UD; Alcon Laboratories, Inc.). The artificial tears were used every hour during the first day, and five times daily for 1 month. The antibiotic was used every hour for the first day, and then decreased to five times daily for 10 days. The steroid was used every hour for the first day, then decreased to five times daily for 1 week and then decreased gradually over 4 weeks. A systemic analgesic was prescribed to manage pain due to CXL. Patients were instructed to avoid eye rubbing. All patients were examined for a complete ophthalmological assessment, including UCVA, BCVA, refraction, and Scheimpflug imaging at 1,3,6, and 12 months postoperatively (Figure 1). Anterior segment OCT was done only once during follow-up
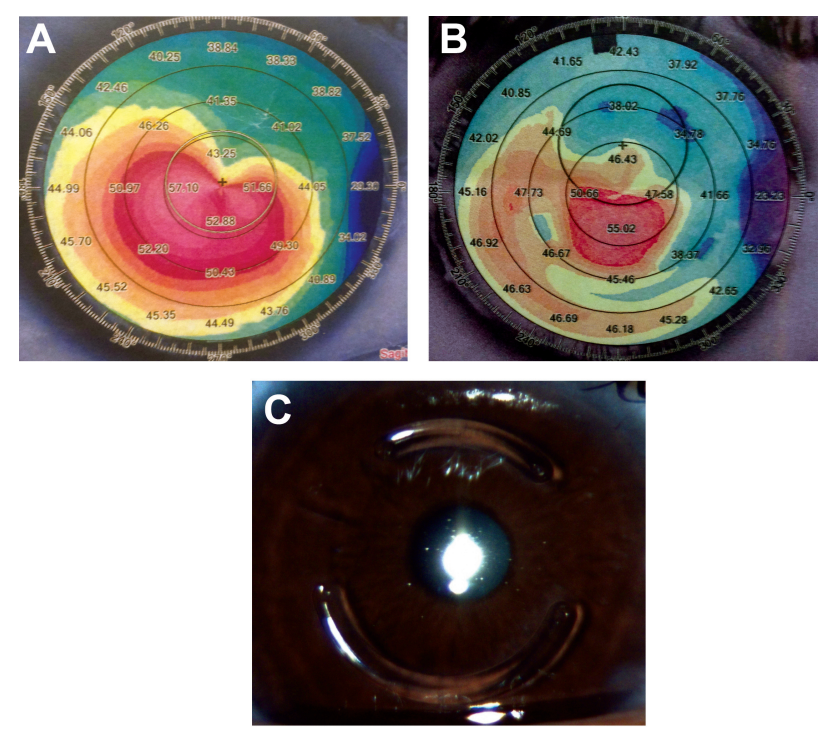

Figure I The preoperative (A) and I-year postoperative (B) appearance of the sagittal anterior map of the Sirius Scheimflug imaging camera (CSO, Italy) of one patient's right eye after implantation of two Keraring segments (C) followed by transepithelial accelerated corneal collagen cross-linking. at 1 month to detect ICRS depth and position and the corneal demarcation line of cross-linking. All complications, if any, were documented.

\section{Statistical analysis}

Data were analyzed using SPSS computer program version 22.0. Quantitative data were expressed as means $\pm \mathrm{SD}$, median and range. Qualitative data were expressed as number and percentage. The data were tested for normality using Kolmogrov-Smirnov test and Shapiro-Wilk test. The nonparametric Wilcoxon test was used for data that were not distributed normally. Paired Samples $t$-test was used for normally distributed data. A 5\% level was chosen as a level of significance in all statistical tests used in the study.

\section{Results}

Eighteen eyes of 11 children ( 9 males and 2 females) with VKC and progressive keratoconus were included in this prospective non-comparative study. The age range was 10-17 years with a mean \pm SD of $13.6 \pm 2.5$ years. Seven cases had bilateral treatment while the other four cases had a unilateral treatment.

Marked improvement was noticed in all patients 1 year postoperatively; mean \pm SD UCVA improved from $1.01 \pm 0.2$ to $0.6 \pm 0.2$ ( $\log \mathrm{MAR})$ while the BCVA improved from $0.6 \pm 0.2$ to $0.4 \pm 0.2$. All keratometry values showed significant decrease 1 year postoperatively: flat $\mathrm{K}$ decreased from $47.9 \pm 2.6 \mathrm{D}$ to $44.7 \pm 2.2 \mathrm{D}$, steep K decreased from $52.8 \pm 3.1 \mathrm{D}$ to $47.7 \pm 3.5 \mathrm{D}$ while the average $\mathrm{K}$ decreased from $50.3 \pm 2.7 \mathrm{D}$ to $45.8 \pm 3.1 \mathrm{D}$ (Figure 2).

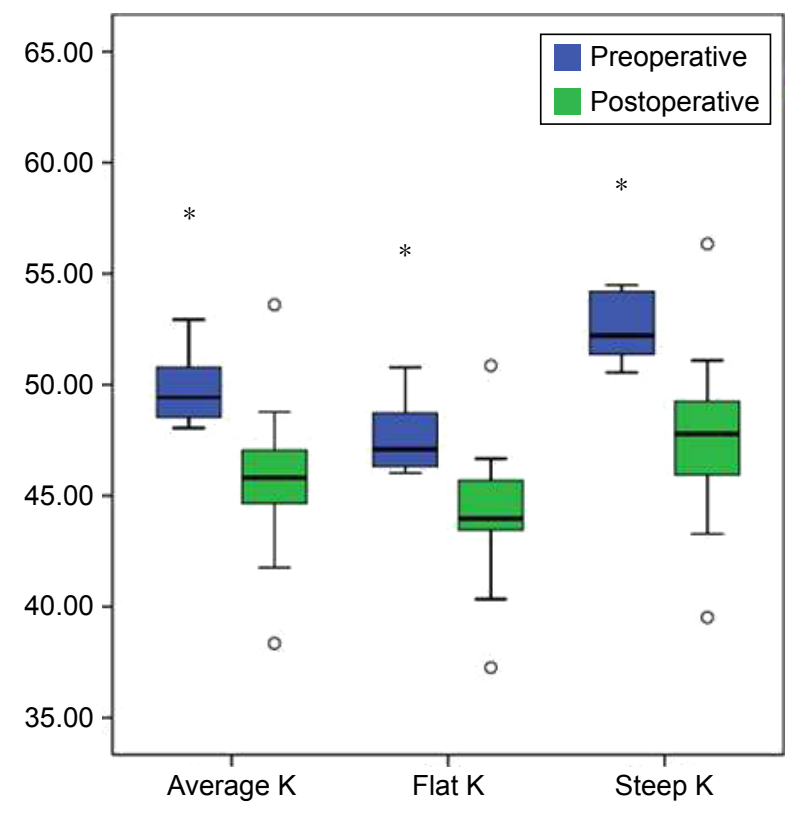

Figure 2 Box blot comparison of average $K$, flat $K$, and steep $K$ pre and postoperatively in the study patients. ${ }^{*} p$-value is less than 0.001 . 
As regards refraction, the mean $\pm \mathrm{SD}$ manifest sphere was reduced from $-5.8 \pm 4.4 \mathrm{D}$ to $-1.9 \pm 3.07$, manifest cylinder decreased from $-7.8 \pm 2.1 \mathrm{D}$ to $-2.6 \pm 1.2 \mathrm{D}$ while the mean spherical equivalent reduced from $-9.4 \pm 4.8 \mathrm{D}$ to $-3.1 \pm 3.4 \mathrm{D}$.

The mean thinnest location decreased from $454.4 \pm 33.6 \mu \mathrm{m}$ to $440 \pm 30.9 \mu \mathrm{m}$. All the parameters showed statistically significant change postoperatively with a $P$-value $<0.001$ and $\mathrm{n}=18$.

No intraoperative complications were reported, except for difficult insertion in one case and suction loss in another one. Postoperatively, no keratitis, extrusion, migration, vascularization, or stromal necrosis was noted. Also, no postoperative infection or corneal melting occurred during follow-up in any of the cases. However, all cases suffered from recurrent attacks of activity of VKC during the 1 year follow-up period, and all were successfully managed with topical steroids without adverse effects on their ICRS.

\section{Discussion}

$\mathrm{VKC}$ is a common cause of kertoconus in our locality. Many ophthalmologists discourage the use of intrastromal corneal rings in these patients because of the high risk of disease progression and the increased lability of complications especially resulting from persistent eye rubbing.

This study assessed the safety and efficacy of femtosecond laser-assisted Keraring implantation followed after 2 weeks by transepithelial accelerated CXL in children with keratoconus and VKC and found significant improvements in UCVA, BCVA, manifest refraction, and keratometric values which remained stable over a period of 1 year.

Although many studies evaluated sequential ICRS and TE-CXL and many others assessed the treatment of pediatric keratoconus, to the best of our knowledge, this study is the first one that addresses management of keratoconus in children with VKC.

Abdelmassih et a ${ }^{15}$ retrospectively evaluated ICRS followed after 1 month by cross-linking in 12 children (17 eyes) with keratoconus and found marked improvement in UDVA and CDVA with the reduction in keratometry and spherical equivalent which were stable through relatively long duration of 4 years. The only complication reported in this study was removal of one ring segment after 2 years due to vascularization and corneal thinning.

Elsaftawy et a ${ }^{16}$ assessed sequential manual Keraring implantation and transepithelial collagen cross-linking in adult keratoconus. They operated on 40 eyes that were divided into two groups with the first group having Keraring implantation only while the second group having Keraring implantation followed by TE-CXL after 1 month and concluded that Keraring implantation is an effective technique for keratoconus but that the addition of TE-CXL has the advantages of halting the progression of the disease and providing more improvements in spherical refraction.

Similar to our study, Artola et a $1^{17}$ tested the efficacy of the accelerated transepithelial cross-linking technique using the Avedro KXL system in 19 eyes of 12 adult patients and followed them for 1 year. They found that the mean depth of the corneal demarcation line measured by OCT was $205.19 \pm 19.75 \mu \mathrm{m}$ with a range of $153-203 \mu \mathrm{m}$ and concluded that accelerated transepithelial cross-linking may be a useful technique for the management of progressive keratoconus because it can stabilize the topographic and aberrometric profile of the cornea for a period of 1 year.

Although our study differs from these studies as regards the sample of patients included and the procedures done, the results are comparable as regards safety and efficacy.

A shortcoming of this study is the lack of a control group of patients with VKC and keratoconus but without having any intervention or having cross-linking only to isolate the effects of Keraring implantation in these patients. Parental education about the hazards of eye rubbing on the ICRSs and its role in the pathogenesis and progression of keratoconus as well as the prompt management of the recurrent attacks of VKC is crucial to the success of treating their children's disease.

In conclusion, the success rate and safety of sequential femtosecond laser-assisted Keraring implantation and transepithelial accelerated cross-linking in children with keratoconus and vernal keratoconjunctivits are comparable to those seen in adults and in children without VKC. However, studies with larger samples and longer follow-up periods are needed. Also, children with severe form of VCK need further studies.

\section{Disclosure}

The author reports no conflicts of interest in this work.

\section{References}

1. Krachmer JH, Feder RS, Belin MW. Keratoconus and related noninflammatory corneal thinning disorders. Surv Ophthalmol. 1984;28: 293-322.

2. Rabinowitz YS. Keratoconus. Surv Ophthalmol. 1998;42:297-319.

3. Kennedy RH, Bourne WM, Dyer JA. A 48-year clinical and epidemiologic study of keratoconus. Am J Ophthalmol. 1986;101:267-273.

4. Bonini S, Coassin M, Aronni S, Lambiase A. Vernal keratoconjunctivitis. Eye. 2004;18:345-351.

5. Bremond-Gignac D, Donadieu A, Leonardi A, et al. Prevalence of vernal keratoconjunctivitis: a rare disease? Br J Ophthalmol. 2008;92: 1097-1102. 
6. Wollensak G, Spoerl E, Seiler T. Stress-strain measurements of human and porcine corneas after riboflavin-ultraviolet-A-induced cross-linking. $J$ Cataract Refract Surg. 2003;29:1780-1785.

7. Goldich Y, Marcovich AL, Barkana Y, Avni I, Zadok D. Safety of corneal collagen cross-linking with UV-A and riboflavin in progressive keratoconus. Cornea. 2010;29:409-411.

8. Magli A, Forte R, Tortori A, Capasso L, Marsico G, Piozzi E. Epitheliumoff corneal collagen cross-linking versus transepithelial cross-linking for pediatric keratoconus. Cornea. 2013;32(5):597-601.

9. Colin J, Cochener B, Savary G, Malet F. Correcting keratoconus with intracorneal rings. J Cataract Refract Surg. 2000;26(8):1117-1122.

10. Chan CC, Sharma M, Wachler BS. Effect of inferior-segment Intacs with and without C3-R on keratoconus. J Cataract Refract Surg. 2007; 33(1):75-80.

11. El-Raggal TM. Sequential versus concurrent KERARINGS insertion and corneal collagen cross-linking for keratoconus. Br J Ophthalmol. 2011;95(1):37-41.

12. El Awady H, Shawky M, Ghanem AA. Evaluation of collagen crosslinking in keratoconus eyes with Kera intracorneal ring implantation. Eur J Ophthalmol. 2012;22(Suppl 7):S62-S68.
13. El-Raggal TM. Effect of corneal collagen crosslinking on femtosecond laser channel creation for intrastromal corneal ring segment implantation in keratoconus. J Cataract Refract Surg. 2011;37(4):701-705.

14. Coskunseven E, Jankov MR II, Hafezi F, Atun S, Arslan E, Kymionis GD. Effect of treatment sequence in combined intrastromal corneal rings and corneal collagen crosslinking for keratoconus. J Cataract Refract Surg. 2009;35:2084-2091.

15. Abdelmassih Y, El-Khoury S, Dirani A, et al. Safety and efficacy of sequential intracorneal ring segment implantation and cross-linking in pediatric keratoconus. Am J Ophthalmol. 2017;178:51-57.

16. Elsaftawy HS, Ahmed MH, Saif MY, Mousa R. Sequential intracorneal ring segment implantation and corneal transepithelial collagen crosslinking in keratoconus. Cornea. 2015;34(11):1420-1426.

17. Artola A, Piñero DP, Ruiz-Fortes P, Soto-Negro R, Pérez-Cambrodí RJ. Clinical outcomes at one year following keratoconus treatment with accelerated transepithelial cross-linking. Int J Ophthalmol. 2017;10(4): $652-655$.
Clinical Ophthalmology

\section{Publish your work in this journal}

Clinical Ophthalmology is an international, peer-reviewed journal covering all subspecialties within ophthalmology. Key topics include: Optometry; Visual science; Pharmacology and drug therapy in eye diseases; Basic Sciences; Primary and Secondary eye care; Patient Safety and Quality of Care Improvements. This journal is indexed on

Submit your manuscript here: http://www.dovepress.com/clinical-ophthalmology-journal

\section{Dovepress}

PubMed Central and CAS, and is the official journal of The Society of Clinical Ophthalmology (SCO). The manuscript management system is completely online and includes a very quick and fair peer-review system, which is all easy to use. Visit http://www.dovepress.com/ testimonials.php to read real quotes from published authors. 